\title{
Hybrid Data Filtering Approach for Mobile Wireless Sensor Networks
}

\author{
Kavita Gupta \\ Research Scholar, \\ Maharishi Markendeshwar University, \\ Mullana
}

\author{
Aarti Singh \\ Associate Professor, \\ Maharishi Markendeshwar University, \\ Mullana
}

\begin{abstract}
This paper presents a hybrid data filtering approach for mobile wireless sensor networks. This hybrid approach ensures filtering of data before it is being sent to sink node from the sensor network. Further, this approach reduces amount of noisy data being transferred, thereby increasing network lifetime and reducing rate of packet transmission in the network. However, slight increase in packet transmission time has been observed which is acceptable considering the improvement in quality of data being received. Present approach has been implemented, however its comparison with other existing approaches is still left as future work.
\end{abstract}

\section{Keywords}

Data Filtering, Hybrid Filtering, Energy efficiency, Periodic Filtering, noisy data.

\section{INTRODUCTION}

In field of Mobile Wireless Sensor Networks (MWSNs) nodes are autonomous and have self healing and self organizing capability. In these networks energy efficiency plays an important role. Communication among these sensor nodes consumes major amount of energy, since nodes in these networks have limited amount of energy thus developing sustainable and energy efficient sensor network is open research challenge. Literature $[1,2,3]$ reveals that clustering and message filtration are two processes that can reduce the energy consumption level in MWSN communications. Aggregating from various sensor nodes involves redundant data or vague information. Thus advanced algorithms $[4,5,6]$ needs to be applied for removing redundant information for getting the optimum information. These advanced algorithms are classified in terms of filtration process being deployed in them for noise removal. After noise removal filtered data is forwarded to base station, here noise is specified as a kind of disturbance that arises due to environmental conditions like temperature, humidity, pressure etc. Filtering is the process of removing the non-required information from the bulk of received information and providing the smooth relevant data. In filtering process various filter are used differently depends upon their area of application but all of them works on same working principle i.e. they all process the sensed data and extract the relevant information. Various statistical and mathematical models are used for data filtration process. Most popular types of filters are Bloom filter, Mobile filter, Enroute filter to name a few, where:

- Bloom Filtering [4]: Bloom filter is a space efficient filtering scheme that maintains data structure suitable for bulky data where hashing mechanism is used for data accessing.

- Particle Filtering [5]: Sensor location is identified using self localization algorithms and using these location parameters remaining sensor nodes are estimated by using particle filters.

- En-route Filtering [6]: Randomized key generation process is used for identifying the unused data. This filtering mechanism is suitable for data filtering and protecting data against various security attacks.

Filtering is applied at various levels of clustered wireless sensor networks to filter the redundant information and forward the processed data to cluster head and then to the base station, thereby improving the quality of information received from this network. Filtering approach can be categorized in three ways as Demand driven, periodic and hybrid. In demand driven approach filtering process is executed for filtering the required data as per user demand. In Periodic filtering, filtering task is performed after fix time interval and Hybrid filtering is the combination of above two approaches. Previous studies reveal that clustering in sensor networks offers the energy efficient sensor networks and prolongs the network life time. In our previous work, Energy Efficient Cluster Head election [1] mechanism along with data aggregation \& result sharing mechanism [20]

However, filtration of noise from the useful information was left intentionally and would be taken up in this work. This work deploy dynamic filters in hybrid filtering strategy for accomplishing the filtering process and forwards updated copy of data to sink, thereby reducing network traffic to some extent.

Next section explores literature in the related domain.

\section{RELATED WORK}

Various researchers have done tremendous work in the field of energy efficient sensor networks and number of filtering mechanisms has already been proposed. Non linear extended Kalman filter [7] uses interlacing multiple model (IMM) for tracking mobile stations. Coordinated turn model is used to improve the tracking performance of proposed scheme.

False report detection and dropping mechanism proposed by [6] uses key based authentication code attached with each event report. Each node verifies the authentication code that was attached with progressed report gives efficient results in terms of reducing the energy consumption level and dropping out bogus reports but still there are chances of data loss at compromised node. This scheme is not suitable for shared data nodes.

Bandwidth efficient Cooperative Authentication (BECAN) [8] scheme is used to detect injected false data in sensor field. ID based authentication scheme is proposed that allows batch verification of multiple signatures for identifying the false injection of data. It has been observed that ID based scheme offers good results as compare to BECAN but ID generation and assigning to all nodes is a time consuming process. 
Cluster based false data filtering scheme [9] works on early stage of data collection and identifies the false data earlier thus saves energy. Rekey management technique is used to identifythe injected false data and improves the system life time. It also increases the security of the network however in case of cluster head failure results are not as per expectations.

Command based filtering mechanism [10] is designed here for accepting and rejecting the human interventions by making the use of Petri nets. Petri nets approach is used to analyze the operated behavior and commands for synthesizing the filters, request for stopping the unrestricted commands and command supervision events needs to be handled separately in different environments.

Use of Kalman filtering for under water sensors using cross layer and time synchronization is proposed by [11]. It controls the Doppler shift caused due to mobility of sensor nodes. This scheme offers optimized results in terms of accuracy and energy efficiency. However, its practical implementation for under water environment is left as future work.

Event driven approach proposed by Xiaohua and Qing [12] is used to analyze the sensor behavior for data transmission over the network. Transmission behavior of each sensor node is analyzed on the basis of its filtering error measurement. Performance of proposed mechanism is analyzed on basis of various filtering parameters. Each sensor node transfers the data packet only when its local error value exceeds the threshold value.

Nonlinear filtering mechanism using channel fading is being proposed by [13] that ensure the stability of Unscented Kalman Filter (UKF). Due to channel fading there are chances of transmission failure and fluctuation of signal. This proposed approach reflects that channel gain will be uncorrelated over the time and space.

Effective signal to noise ratio estimation using kalman filter is proposed by [14] that includes signal to noise ratio along with link quality indicator. This proposed approach reduces the energy consumption level and utilize the full capacity of all channels that will further improve the accuracy of estimation. But the method proposed for link quality indicator computation is not accurate in terms of link margins, because it takes only few inputs for link quality indicator estimation and ignored some important parameters.

Localization based data filtering mechanism proposed by [15] estimates the location of sensor using filtering mechanism. An Eigen vector based approach is offered to estimate the location of sensor where position is specified as the integration of various positional parameters and acceleration data values. Major hurdle for implementation of this method is that positional parameters need to be highly accurate for getting the efficient results.

GRPEF (Grouping-Enhanced Resilient Probabilistic En-Route Filtering) [16] is an efficient distributed algorithm uses location aware scheme for detecting nodes without splitting them in extra grouping that will further improve the network efficiency and reduce the energy consumption level.

Prediction based data transportation scheme proposed by [17] uses partial omission of network for improving the network life time, reduces network traffic and the energy consumption levels.

Application based Extended kalman filtering approach is used by [18] for evaluating efficient information processing by making the use of extended kalman filtering in sub surfaces as kalman filter can be applicable in both theory and practical and it also reduces the level of errors.

From above literature review it's clear that filtering noisy data from sensed data is desired by research community and thus many efforts are being made in this direction. However, till date no filtering mechanism has been accepted as standard and attention needs to be paid in this direction. Next section elaborates our proposed mechanism.

\section{PROPOSED WORK}

In present work MWSN nodes are arranged in multilevel clustered network. In this clustered network data collection process is executed by cluster head, each node sends its data to its cluster head $(\mathrm{CH})$ that will further forward the data toits base station. Cluster Head $(\mathrm{CH})$ is responsible for data collection, aggregation of data and its filtering before passing data to base station. Data aggregation scheme [20] introduced mobile agents for executing the data aggregation process followed by result sharing. This aggregated data would certainly contain some redundant data and noisy information that must be removed as it would increase the size of data and would lead to unnecessary energy consumption while transmission.

This work proposes hybrid approach for data filtering that is being applied on the aggregated data for removing redundancy and noise. This approach amalgamates demand driven and periodic filtering strategies in one mechanism making it hybrid. Hybrid filtering approach can ensure that sensed data always gets filtered before being sent to base station. Static as well as dynamic filters have been deployed in it for filtering of noisy and redundant data. This mechanism deploys intelligent agents for data filtering, termed as Filtering Agent $\left(\mathrm{FA}_{\mathrm{i}}\right)$. These FAs are deployed on cluster heads $(\mathrm{CH})$ at various levels. All the cluster heads at the same level execute result sharing module and send their aggregated filtered data to next higher level cluster head where again filtering of data takes place and this finally filtered copy of data is forwarded to base station ultimately. Filtration processes depicted in the figure 1.1. This approach filters data using two cases

i. In Periodic filtering data filtering process is executed after a fixed time interval.

ii. In demand driven filtering data filtration process is executed as per user requirement.

Since sensors are deployed in hostile environment they sense different type of data depending on the capability of sensor node. Nature and size of data being sensed will be different for different sensors and will depend on parameter being sensed such as temperature, pressure etc. Let $\mathrm{K}$ be the size of data received at sink node, $n$ be number of data packets received and $k$ is the size of each data packet, therefore

$$
K=n * k
$$

In case 1 mentioned above periodic data filtering takes places using algorithm 1.3, thus filtering of data is done on periodic basis, after each periodic cycle data size is computed and we select first $\mathrm{K}$ bits of filtered data for transferring to next higher level. In second case filtering approach is demand driven, where data is filtered on the basis of specified value for which binary search is being applied. Data $\log$ is maintained as per table 1.1 for sensor data where various parametric values are arranged in sorted manner. 


-

$\mathrm{P}(\mathrm{n})$

Table 1.1 Data log on the basis of parameters

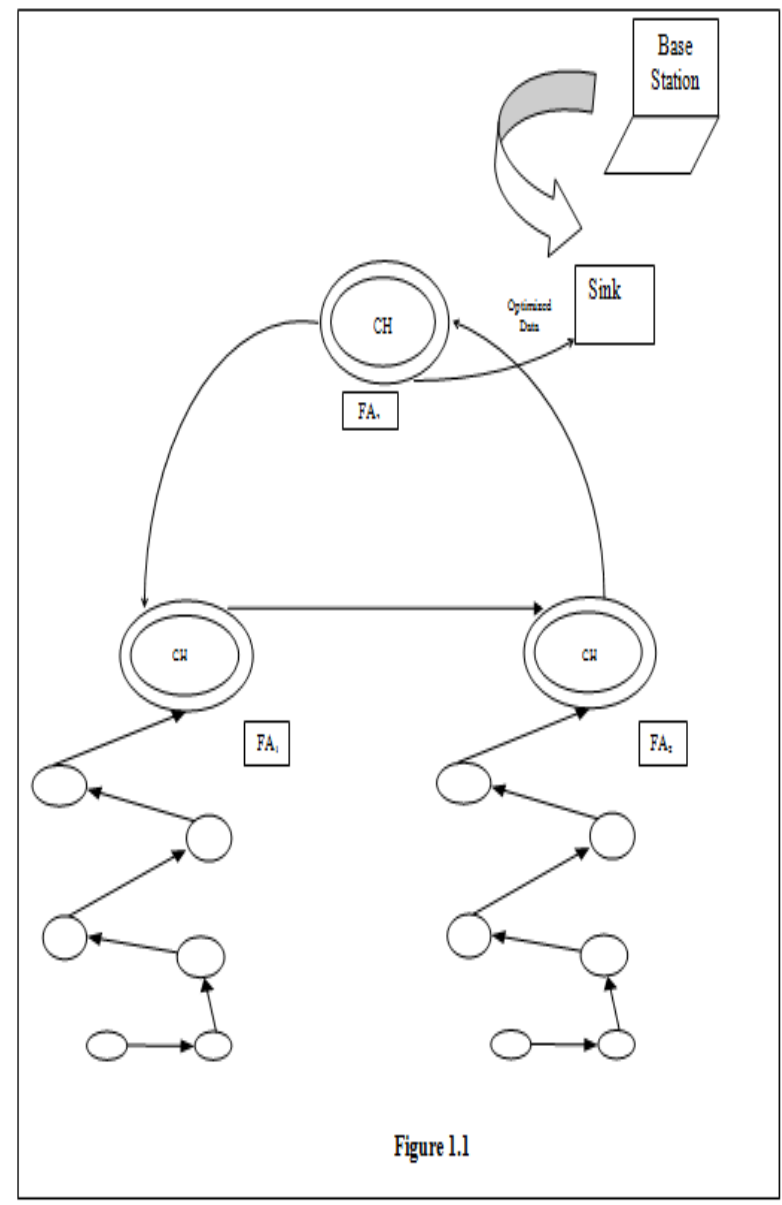

\section{RESULT \& DISCUSSION}

This proposed hybrid filtering mechanism has been implemented on MATLAB. Using this mechanism delay in packet transmission gets increased because filtering the sensed data consumes some additional time as compared to without data filtering. However, amount of noise in data gets reduced significantly. Further, rate of energy consumption also gets reduced since now only filtered data is getting transferred from one level to other, thereby reducing transfer of unnecessary packets in the MWSNs. Figures 1.2-1.4 present implementation results.

Algorithm 1.3 datafilterig ()
choose (case)
Store filtered data
exit()

\section{Case 1: Data filtering in periodic manner}

Node Deployment process

Call algorithm Cluster head Selection()

Call algorithm Data Aggregation()

Data size is fixed to $K$ bits, $\max ($ datasize $)=\mathrm{K}$

Datasize(j)

$=\sum \operatorname{size}(p(1)), \operatorname{size}(p(2)), \ldots \ldots ., \operatorname{size}(p(n))$

Step 6: if datasize $(j)>K$, then datasize $(j)$

$$
=\max (\text { (datasize })
$$

\section{Case 2: Data filtering on Demand Driven}

Node Deployment process

Call algorithm Cluster head Selection()

Call algorithm Data Aggregation()

Data size is fixed to $K$ bits, $\max ($ datasize $)=\mathrm{K}$

Datasize $(K)$

$=\sum \operatorname{size}(p(1)), \operatorname{size}(p(2)), \ldots \ldots ., \operatorname{size}(p(n))$

Call binary search ( )

return data .

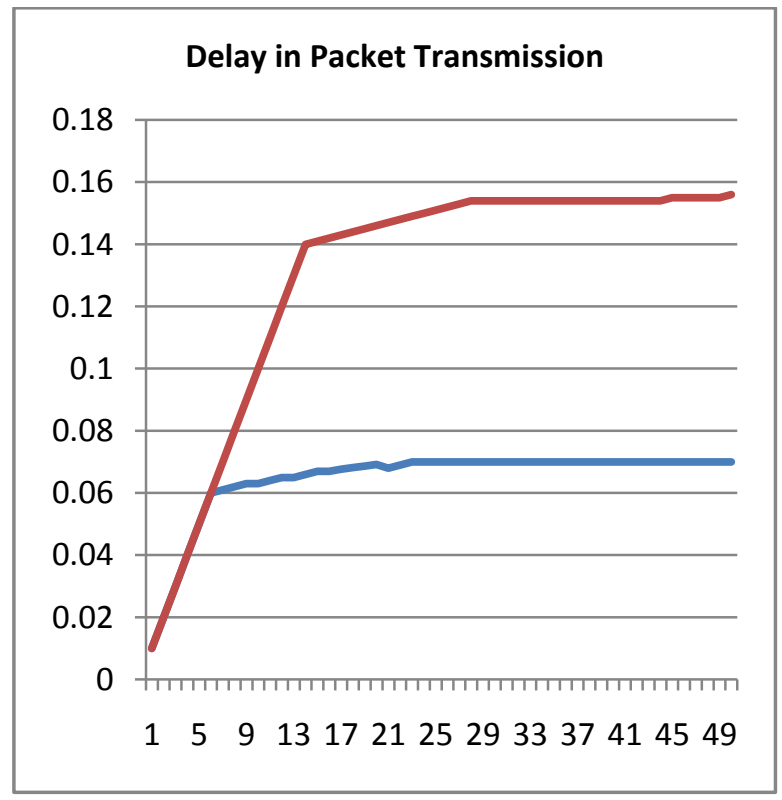

Fig 1.2 


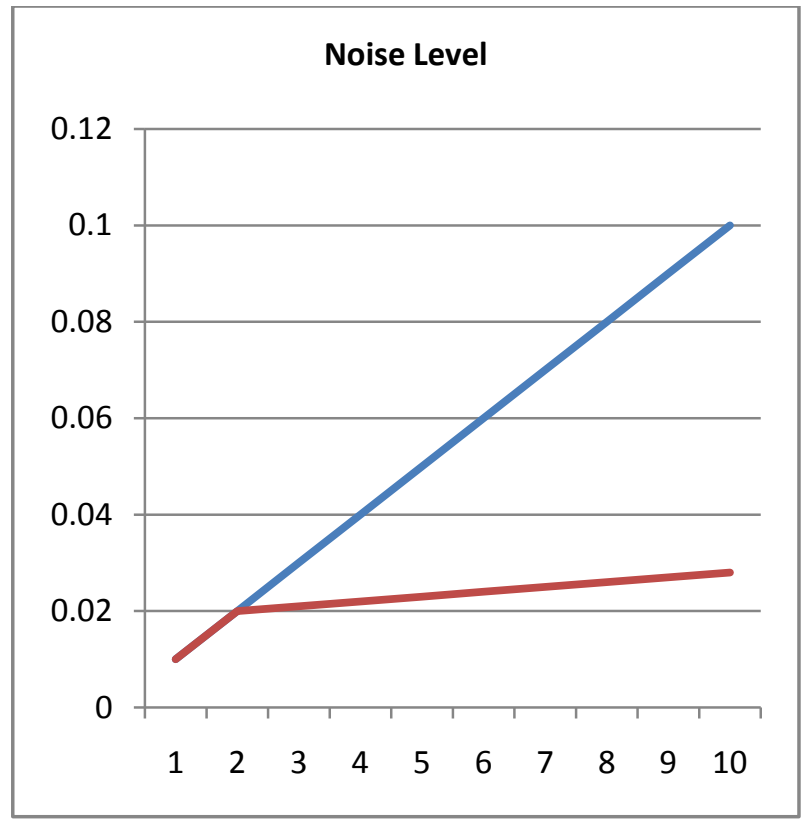

Fig 1.3

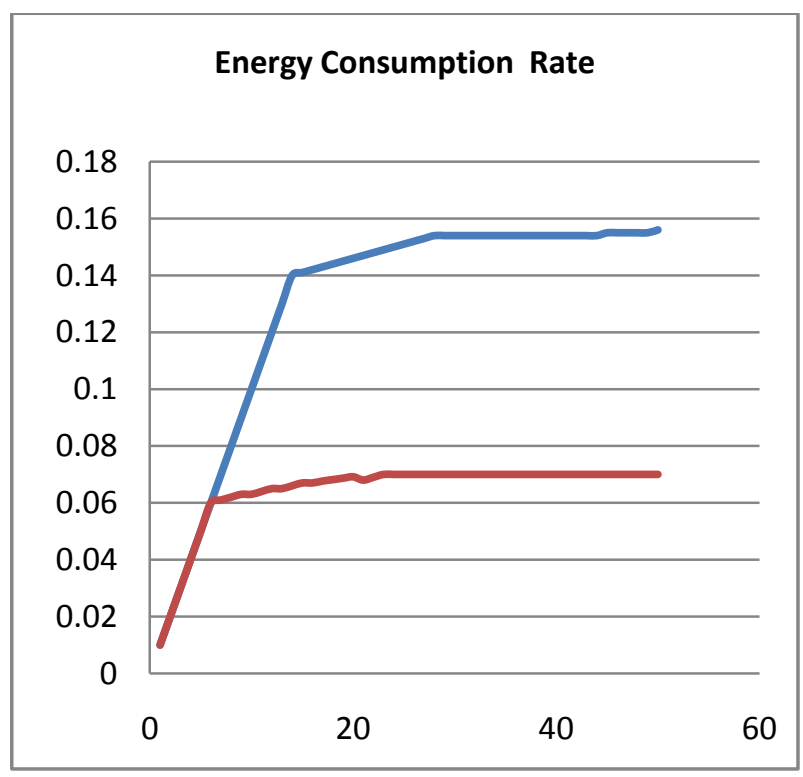

Fig 1.4

\section{REFERENCES}

[1] Gupta K., Singh A., Singh R., Mukherjee S. 2015. An Improved Cluster Head Selection Algorithm for Mobile Wireless Sensor Networks. Journal of Network Communications and Emerging Technologies.

[2] Anitha, R.U., Kamalakkannan, P. 2013. Energy efficient cluster head selection algorithm in mobile wireless sensor networks, In Proceeding of International Conference on Computer Communication and Informatics (ICCCI).

[3] Gupta, D., Verma, R., 2014. An enhanced cluster-head selection scheme for distributed nheterogeneous wireless sensor network. I proceedings of International Conference on Advances in Computing, Communications and Informatics (ICACCI).
[4] Reinhardt A., Morar O., Santiniy S., Z"oller S. and Steinmetz R.. 2012. CBFR: Bloom Filter Routing with Gradual Forgetting for Tree-structured Wireless Sensor Networks with Mobile Nodes In Proceedings of $13^{\text {th }}$ International Symposium of World of Wireless, Mobile and Multimedia Networks (WoWMoM).

[5] Hanselmann T., Zhangy Y., Morelande M., MohdIfranMd Nor, Jonathan Wei Jen Tan, Xing-She Zhouy, Yee Wei Law. 2010. Self-Localization in Wireless Sensor Networks Using Particle Filtering with Progressive Correction. In proceedings of $5^{\text {th }}$ International Conference on Communication and Networking.

[6] Ye F., Luo H., Zhang S. 2005. Statistical En-Route Filtering of Injected False Data in Sensor Networks. IEEE Journal On Selected Areas in Communications.

[7] Chang D. Fang M. 2014. Bearing-Only Maneuvering Mobile Tracking With Nonlinear Filtering Algorithms in Wireless Sensor Networks. IEEE System Journal.

[8] Thampi A. K.G, Nithya L.M. 2011. An Efficient IDBased Scheme for Filtering Gang Injected False Data in Wireless Sensor Networks. International Journal of Advanced Research in Computer and Communication Engineering.

[9] Narayanan U., Soman A. 2013. CAFS: Cluster based Authentication scheme for Filtering False data in wireless Sensor network. International Journal of Advanced Research in Computer and Communication Engineering.

[10] Jin Shyan Lee. 2008. A Petri Net Design of Command Filters for Semiautonomous Mobile Sensor Networks. IEEE Transactions On Industrial Electronics.

[11] Liu J., Zuba M., Peng,mber Z., Cui J., Zhou S. 2014. DA-Sync: A Doppler-Assisted Time-Synchronization Scheme for Mobile Underwater Sensor Networks. IEEE Transactions On Mobile Computing.

[12] XiaohuaGe, Han Q. 2014. Distributed event-triggered H1 filtering over sensor networks with communication delays. Information Sciences Journal of Elsevier.

[13] Li Li, Xia Y. 2015. UKF-based nonlinear filtering over sensor networks with wireless fading channel. Information Sciences Journal of Elsevier.

[14] Qin F., Dai X., Mitchell J. 2013. Effective-SNR estimation for wireless sensor network using Kalman filter. Ad-hoc Network Journal of Elsevier.

[15] Hur H., Ahn H. 2012. Discrete-Time Ho Filtering for Mobile Robot Localization Using Wireless Sensor Network. IEEE Sensor Journal.

[16] Li J., Yu L., Gao H., Xiong S. 2012. Grouping-Enhanced Resilient Probabilistic En-Route Filtering of Injected False Data in WSNs. IEEE Transactions On Parallel and Distributed Systems.

[17] Blaß E., Tiede L., Zitterbart M. 2006. An EnergyEfficient and Reliable Mechanism for Data Transport in Wireless Sensor Networks. In Proceedings of International Conference on Networked Sensing Systems. 
International Journal of Computer Applications (0975 - 8887)

Volume 138 - No.8, March 2016

[18] Juneja D., Sharma A., Sharma A.K. 2011. A Novel Application of Extended Kalman Filter for Efficient Information Processing In Sub surfaces. International Journal of Computer Applications.

[19] Juneja D., Gupta K., Singh S. 2015. Exploiting Mobility of Agents for Data Sharing and Aggregation in a
Clustered Mobile Wireless Sensor Networks. Journal of Network Communications and Emerging Technologies.

[20] T. Rappaport 1996. Wireless Communications: Principles \& Practice .Englewood Cliffs, NJ: PrenticeHall. 